\title{
Hypofractionation in prostate cancer radiotherapy: a step forward towards clinical routine
}

\author{
Barbara Vischioni, Rachele Petrucci, Francesca Valvo \\ Radiation Oncology Department, CNAO National Center for Oncological Hadrontherapy, Pavia, Italy; \\ Correspondence to: Barbara Vischioni. Radiation Oncology Department, CNAO National Center for Oncological Hadrontherapy, Strada Campeggi \\ 53, 27100 Pavia, Italy. Email: vischioni@cnao.it. \\ Provenance: This is an invited article commissioned by the Section Editor Dr. Xiao Li, MD (Department of Urology, Jiangsu Cancer Hospital \& \\ Jiangsu Institute of Cancer Research \& Nanjing Medical University Affiliated Cancer Hospital, Nanjing, China). \\ Comment on: Bruner DW, Pugh SL, Lee WR, et al. Quality of life in patients with low-risk prostate cancer treated with hypofractionated vs \\ conventional radiotherapy: a phase 3 randomized clinical trial. JAMA Oncol 2019;5:664-70.
}

Submitted Oct 20, 2019. Accepted for publication Nov 06, 2019.

doi: $10.21037 /$ tau.2019.11.06

View this article at: http://dx.doi.org/10.21037/tau.2019.11.06

Radiotherapy (RT) has always been considered one of the most important curative treatment modalities for prostate tumors along with surgery. The debate in prostate cancer treatment has recently focused on hypofractionated RT (HRT), which consists of treatment schedules of shorterthan-standard duration, and delivered with larger fractionsize to reach the same biological equivalent dose (BED) as conventional RT (CRT) on the prostate tumor target.

Prostate cancer is regarded as relatively radioresistant to CRT. Laboratory experiments and clinical practice have shown that prostate $\alpha / \beta$ ratio is low (range of $1.5-3 \mathrm{~Gy}$ ), therefore fewer large-dose fractions have been employed in HRT regimens instead of the dose escalation of CRT with 2 Gy daily fractions (1). In this regard, since the fractionsize sensitivity is significantly higher for prostate cancer than for nearby dose-limiting normal tissues, larger dose fractions are supposed to increase tumor control probability, without increasing toxicity. Moderate HRT is usually delivered in 19 to 28 fractions of 2.5 to 3.4 Gy per fraction. Three recent phase III non-inferiority randomized trials have shown the same efficacy for moderate HRT compared to CRT, but different results in toxicity (2). Besides the RTOG 0415 trial discussed first in Lee et al. (3), and, further, in Bruner et al. (4), two other recently published phase III randomized trials have demonstrated that HRT is well tolerated $(5,6)$, although with a moderate increase in toxicity when compared to CRT schedules. In the Dutch HYPRO trial randomizing 820 men with predominantly high-risk prostate cancer to either 78 Gy in 39 fractions or $64.6 \mathrm{~Gy}$ in 19 fractions, gastrointestinal toxicity $\geq$ grade 2 up to 120 days post-RT was more common in HRT arm compared to CRT, but no difference between arms was recorded by 18 weeks after the start of RT (7). Improvements in biochemical disease-free survival outcome has not been reported so far in these trials for moderate HRT over CRT, although possibly longer follow up is needed to assess outcome endpoint after RT. Following these recent reports, the American Society for Radiation Oncology (ASTRO), American Society of Clinical Oncology (ASCO) and American Urological Association (AUA), have strongly recommended, in their 2018 joined evidence-based clinical practice guidelines, the adoption of moderate HRT in men with low- and intermediate-risk prostate cancer receiving external beam RT to the prostate as alternative to active surveillance (8).

The work of Bruner et al. (4) completes the report on clinical outcome results of the phase III trial RTOG 0415, which concludes that disease-free survival after HRT was not inferior to CRT in men with low-risk prostate cancer. Concerning late toxicity, gastrointestinal and genitourinary physician-reported adverse events were higher in patients who received curative HRT compared to CRT (3). Patients in the trial were randomly assigned to two different treatment arms, one of CRT up to total dose of 73.8 Gy in 41 fractions delivered over 8.2 weeks, and another of HRT of 70 Gy in 28 daily sessions delivered over 5.6 weeks. In 
order to further support the adoption of HRT, since the concern of slight increase in late toxicity for the HRT arm compared to the CRT arm, the study by Bruner $e t$ al. addresses differences in several aspects of quality of life (QOL) or subjective parameters among the patients enrolled in the RTOG 0415 randomized trial, by using several questionnaires administered at different time points during follow up, measuring bowel, urinary, sexual and hormonal domains with the Expanded Prostate Index Composite questionnaire (EPIC), anxiety and depression with the 25item Hopkins Symptoms Checklist (HSCL), and global QOL with the EuroQoL-5 Dimension Questionnaire (EQ-5D). A total of 962 patients enrolled in the trial where asked to report on their symptoms and treatment perceptions by completing the QOL questionnaires. QOL data were collected for 478 men from the CRT group and 448 men from the HRT group, at different time points, at baseline and during follow up, up to 60 months, with change scores compared between arms using the Wilcoxon signed rank test.

Nowadays, the number of trials including QOL assessment as an endpoint to validate a specific treatment schedule is increasing, and more patient-centered endpoints are advocated for clinical trials than in the past (9). In this regard, especially when investigating treatments for prostate cancer, increasing attention is directed to patient-reported outcomes (PRO) for more subjective parameters, such as sexuality, anxiety and depression, more reflective of the patient experience compared to physician-reported adverse events. Differences in perception concerning treatment symptoms as reported by clinicians and self-assessment of health-related QOL in men treated for prostate cancer has been already reported (10), and recording of patient self-reported experience with indicators or surrogate is becoming a priority. Unfortunately, so far there is no other standardized method than validated questionnaires that allows investigators to collect such a big amount of sensitive data to assess very important aspects of QOL, which might encounter sometimes the risk to be of poor quality for patient compliance, both at enrollment and over time. In the study by Bruner et al., patient questionnaires response rate was appropriate and balanced for comparison in the 2 treatment groups at enrollment, although compliance declining over time, forcing the statistical analysis to be corrected for missing data. The same limitation, although less pronounced since the shorter follow up time, was noticed for the phase III "Conventional or Hypofractionated High Dose Intensity Modulated
Radiotherapy in Prostate Cancer (CHHiP) randomized trial", where the UCLA Prostate Cancer Index (UCLAPCI), including Short Form (SF)-36 and Functional Assessment of Cancer Therapy-Prostate (FACT-P), or EPIC and SF-12 QOL questionnaires, were completed at baseline, pre-RT, 10 weeks post-RT, and $6,12,18$, and 24 months post-RT (11). The use of different questionnaires with multiple choices to assess same or different aspects of QOL, not only makes comparison of results among trials uneasy, but also renders statistical analysis complex leading to less clear-cut conclusions. Long-time assessment is important for the evaluation of RT toxicity and tolerability. However, the best timing for the different specific QOL aspects assessment has not been defined yet, in general and for prostate cancer patients undergoing RT. Furthermore, several procedures are currently in practice for QOL and PRO questionnaires administration, including face-to-face interviews with or without psychology background, letters and online questionnaires. Easily accessible consolidated, and consensus-driven, PRO and QOL protocol guidelines for conducting assessment need to be followed within clinical trials to facilitate rigorous collection/reporting of data (12).

In the study by Bruner et al., the important dimension of sexual functioning has been investigated with the EPIC questionnaire, and has led to the assessment of no impact on the patient sexual sphere of the HRT compared to the CRT scheme, confirmed also in the CHHiP trial, although in a different patient population regarding risk stratification (11). Since the cohort of the RTOG 0415 trial includes only lowrisk prostate cancer patients that do not undergo hormonal treatment that might impact by itself on sexual functioning, we believe that this data might be of importance in order to sustain the safety of HRT on the patient sexual sphere, which preservation is of paramount importance in determining patient treatment choice. Concerning the other QOL domains evaluated by the EPIC instrumentbowel, urinary and hormonal-in the RTOG 0415 trial, no major changes were recorded for either CRT or HRT treated patients at six months follow up, but at 12 months, HRT patients reported a larger decline in the bowel domain compared to those who received CRT, although the change was not clinically significant (4). Similarly, for the CHHiP trial no significant differences across treatment regimens could be detected both in the primary endpoint of bowel bother and secondary outcome of sexual and urinary domains at different time points up to 24 months. Also, for the CHHiP trial results at longer follow up of at least 
5 years are awaited to assess patient reported tolerability of the HRT scheme compared to CRT (11).

The noninferior cure efficacy previously reported (2) together with the noninferior prostate cancer specific (bowel, bladder and sexual) and general QOL data for patients treated with HRT compared to CRT shown by Bruner and coworkers, might be of paramount importance in supporting the validation of alternative shorter HRT regimens in clinical practice, in general for prostate cancer patients who decides to undergo treatment instead to be closely followed up. In fact, for low-risk prostate cancer one option instead of surgery or RT is active surveillance (13). Outcome results of several trials nowadays sustain the patient preference for surveillance, which has the advantage to avoid or delay treatment discomfort and functional treatment sequelae. In one of the most mature cohort of patients on active surveillance followed up for a median time of 9 years, the risk of dying from prostate cancer was estimated 10 times lower compared to any other reasons unrelated to the cancer at the prostate (14). Since personal patient preference is becoming an important factor in determining the therapeutic option for localized prostate cancer, it is important to add evidence on sustainable level of QOL in favor of HRT schedules such as in the study by Bruner et al. (4). It is now well accepted that with the adoption of shorter treatment duration HRT schemes, patients might benefit of further advantages that might improve their QOL, such as, for example, reduction of distress and costs that derive from long treatment schedules in RT facilities far from home, sometimes requiring undesired sick leave from usual job occupation. This approach might be economically advantageous also for treatment facilities, which might reduce treatment costs and shorten treatment waiting lists by reducing treatment patient duration. At the moment, D'Amico risk classification (15) guides the choice of best treatment option for patient with localized disease were histopathologic features are heavily guiding choice, but in the future genetic testing $(16,17)$, currently under investigation for the potential of predicting metastatic potential after radical prostatectomy, and more complex radiosensitivity index, might be used as a strategy to better stratify patients according to their genetic features to active treatment or surveillance.

The introduction of the new advanced techniques of intensity-modulated RT combined with image guided RT for more precise treatment set-up verification, has stimulated research towards ultra HRT schemes with overall even shorter treatment time compared to the moderate HRT schemes of the randomized trials discussed here so far (18). Apart from the modern linear accelerator of photon RT, in the recent years the landscape of RT cure for prostate cancer is rapidly changing, also with the introduction of particle therapy. Particles by themselves have favorable physical characteristics that allow to better conform the dose to the prostate and thus reducing dose to surrounding normal organs, in particular the rectum, in order to spare toxicity. So far, neither outcome advantage nor clear benefit in toxicity and patients QOL have been reported for proton RT compared to photon RT (19), and several HRT schemes are currently being investigated with proton therapy against standard proton fractionation, for safety and efficacy in clinical trials (20). In Japan, prostate cancer is currently routinely treated with carbon ion RT, after an experimental phase within several controlled clinical trials carried out since 1995 at the National Institute of Radiological Sciences (NIRS). Carbon ions are particles that possess advantageous physical properties same as protons, but higher radiobiological effectiveness (RBE), resulting from high linear energy transfer (LET) beams, estimated to be approximately threefold higher, also compared to photon RT. Carbon ion RT is usually delivered in HRT schemes since the experiments conducted with neutrons, other high-LET particles as carbon ions, in which larger fraction doses tended to lower RBE for both tumor and normal tissues, but with a slower decline of the RBE of the tumor compared to the RBE of the surrounding normal tissues (21). The excellent results of clinical studies carried out at the NIRS in Japan in prostate cancer with CIRT has led to change dose fractionation schedules towards more hypofractionated schemes, from 20 fractions over 5 weeks to 12 over 3 weeks, within clinical trials that showed competitive 5 -year biochemically relapsefree rates compared to CRT, and no impact of incidence of late adverse events $(22,23)$. At the National Center of Oncological Hadrontherapy (CNAO, Italy), patients with high-risk prostate cancer are currently being treated with $66.4 \mathrm{~Gy}(\mathrm{RBE})$ of CIRT delivered in 16 sessions, analogously to Japanese trials from NIRS, or with a CIRT boost to the whole prostate of $16.6 \mathrm{~Gy}$ (RBE) in 4 fractions, followed by a pelvic photon CRT phase up to $50 \mathrm{~Gy}$ to the pelvic lymph nodes and whole prostate (24).

In conclusion, Bruner et al. adds patient-centered important data on the tolerability of moderate HRT schemes for patients undergoing treatment for low-risk prostate cancer, already benefiting from the advantage of significantly spared treatment time because of the shorter 
HRT duration compared to CRT, whose impact on the general QOL data has not been quantified yet with proper indicators. In the era of participative medicine (2), the results of this paper represent a valid patient decision aid that clinician should keep in mind while making treatment decisions together with patients when proposing different curative approaches along with their effectiveness and toxicity.

\section{Acknowledgments}

None.

\section{Footnote}

Conflicts of Interest: The authors have no conflicts of interest to declare.

Ethical Statement: The authors are accountable for all aspects of the work in ensuring that questions related to the accuracy or integrity of any part of the work are appropriately investigated and resolved.

\section{References}

1. Fowler JF. Biological factors influencing optimum fractionation in radiation therapy. Acta Oncol 2001;40:712-7.

2. Vanneste BG, Van Limbergen EJ, van Lin EN, et al. Prostate Cancer Radiation Therapy: What Do Clinicians Have to Know? Biomed Res Int 2016;2016:6829875.

3. Lee WR, Dignam JJ, Amin MB, et al. Randomized Phase III Noninferiority Study Comparing Two Radiotherapy Fractionation Schedules in Patients With Low-Risk Prostate Cancer. J Clin Oncol 2016;34:2325-32.

4. Bruner DW, Pugh SL, Lee WR, et al. Quality of Life in Patients With Low-Risk Prostate Cancer Treated With Hypofractionated vs Conventional Radiotherapy: A Phase 3 Randomized Clinical Trial. JAMA Oncol 2019;5:664-670.

5. Dearnaley D, Syndikus I, Mossop H, et al. Conventional versus hypofractionated high-dose intensity-modulated radiotherapy for prostate cancer: 5 -year outcomes of the randomised, noninferiority, phase $3 \mathrm{CHHiP}$ trial. Lancet Oncol 2016;17:1047-60.

6. Catton CN, Lukka H, Gu CS, et al. Randomized Trial of a Hypofractionated Radiation Regimen for the Treatment of Localized Prostate Cancer. J Clin Oncol 2017;35:1884-90.

7. Incrocci L, Wortel RC, Alemayehu WG, et al.
Hypofractionated versus conventionally fractionated radiotherapy for patients with localised prostate cancer (HYPRO): Final efficacy results from a randomised, multicentre, open-label, phase 3 trial. Lancet Oncol 2016;17:1061-9.

8. Morgan SC, Hoffman K, Loblaw DA, et al. Hypofractionated Radiation Therapy for Localized Prostate Cancer: An ASTRO, ASCO, and AUA EvidenceBased Guideline. J Clin Oncol 2018. [Epub ahead of print].

9. Ramsey S, Schickedanz A. How should we define value in cancer care? Oncologist 2010;15 Suppl 1:1-4.

10. Sonn GA, Sadetsky N, Presti JC, et al. Differing perceptions of quality of life in patients with prostate cancer and their doctors. J Urol 2013;189:S59-65.

11. Wilkins A, Mossop H, Syndikus I, et al. Hypofractionated radiotherapy versus conventionally fractionated radiotherapy for patients with intermediate-risk localised prostate cancer: 2-year patient-reported outcomes of the randomised, non-inferiority, phase $3 \mathrm{CHHiP}$ trial. Lancet Oncol 2015;16:1605-16.

12. Calvert M, Kyte D, Mercieca-Bebber R, et al. Guidelines for Inclusion of Patient-Reported Outcomes in Clinical Trial Protocols: The SPIRIT-PRO Extension. JAMA 2018;319:483-94.

13. Klotz L. Contemporary approach to active surveillance for favorable risk prostate cancer. Asian J Urol 2019;6:146-52.

14. Klotz L, Vesprini D, Sethukavalan P, et al. Long-term follow-up of a large active surveillance cohort of patients with prostate cancer. J Clin Oncol 2015;33:272-7.

15. D'Amico AV. Risk-based management of prostate cancer. N Engl J Med 2011;365:169-71.

16. Klein EA, Cooperberg MR, Magi-Galluzzi C, et al. A 17-gene assay to predict prostate cancer aggressiveness in the context of Gleason grade heterogeneity, tumor multifocality, and biopsy undersampling. Eur Urol 2014;66:550-60.

17. Cuzick J, Stone S, Fisher G, et al. Validation of an RNA cell cycle progression score for predicting death from prostate cancer in a conservatively managed needle biopsy cohort. Br J Cancer 2015;113:382-9.

18. Timon G, Ciardo D, Bazani A, et al. Rationale and protocol of AIRC IG-13218, short-term radiotherapy for early prostate cancer with concomitant boost to the dominant lesion. Tumori 2016;102:536-40.

19. Ishikawa H, Tsuji H, Murayama S, et al. Particle therapy for prostate cancer: The past, present and future. Int $\mathrm{J}$ Urol 2019;26:971-9. 
20. Vargas CE, Schmidt MQ, Niska JR, et al. Initial toxicity, quality-of-life outcomes, and dosimetric impact in a randomized phase 3 trial of hypofractionated versus standard fractionated proton therapy for low-risk prostate cancer. Adv Radiat Oncol 2018;3:322-30.

21. Ando K, Kase Y. Biological characteristics of carbon ion therapy. Int J Radiat Biol 2009;85:715-28.

22. Okada T, Tsuji H, Kamada T, et al. Carbon ion radiotherapy in advanced hypofractionated regimens for prostate cancer: from 20 to 16 fractions. Int J Radiat Oncol

Cite this article as: Vischioni B, Petrucci R, Valvo F. Hypofractionation in prostate cancer radiotherapy: a step forward towards clinical routine. Transl Androl Urol 2019;8(Suppl 5):S528-S532. doi: 10.21037/tau.2019.11.06
Biol Phys 2012;84:968-72.

23. Nomiya T, Tsuji H, Maruyama K, et al. Phase I/II trial of definitive carbon ion radiotherapy for prostate cancer: evaluation of shortening of treatment period to 3 weeks. Br J Cancer 2014;110:2389-95.

24. Marvaso G, Jereczek-Fossa BA, Vischioni B, et al. Phase II multi-institutional clinical trial on a new mixed beam RT scheme of IMRT on pelvis combined with a carbon ion boost for high-risk prostate cancer patients. Tumori 2017;103:314-8. 